\title{
Community Pharmacists' Attitudes and Professional Practice in Relation to the Patient Safety Incidents
}

\author{
Valentina Marinkovic ${ }^{1}$, Tatjana Stojkovic ${ }^{2, \star}$, Milica Zekovic ${ }^{3}$, Ljiljana Tasic ${ }^{1}$, Dusanka Krajnovic ${ }^{1}$ \\ ${ }^{1}$ Department of Social Pharmacy and Pharmaceutical Legislation, Faculty of Pharmacy, University of Belgrade, Belgrade, SERBIA. \\ ${ }^{2}$ Merck Sharp and Dohme Serbia Ltd, Omladinskih Brigada, Belgrade, SERBIA. \\ ${ }^{3}$ Centre of Research Excellence in Nutrition and Metabolism, Institute for Medical Research, University of Belgrade, Belgrade, SERBIA.
}

\begin{abstract}
Background: Medicines dispensing is an error-prone activity, therefore potentially jeopardizing patient safety. This study aimed to assess the community pharmacists' attitudes towards the causes of dispensing errors and preventive measures, as well as their practice in incidents reporting. Materials and Methods: A cross-sectional survey was performed by distributing an adopted and validated questionnaire to a nationwide sample of community pharmacists in Serbia. The questionnaire included sections related to the participants' socio-demographic characteristics, their attitudes towards factors causing dispensing errors and corrective actions, as well as their practice in reporting. Statistical analyses were conducted using SPSS Statistics software ver. 21.0. The associations between categorical variables were analyzed using Chi-square test. Results: The study included 1,004 participants, mainly female $(94.9 \%)$, with the mean age $40.9 \pm 9.9$ years and mean work experience $14.3 \pm 10.0$ years. More than a third of the participants $(35.4 \%)$ indicated an increasing risk of dispensing errors. The main causes included illegible prescriber's handwriting (44.3\%) and interruptions during dispensing $(39.2 \%)$, while the major corrective actions were providing pharmacists with education in clinical pharmacy $(71 \%)$ and reducing the interruptions during dispensing $(63.9 \%)$. The majority of respondents $(85.2 \%)$ stated that they routinely reported dispensing incidents. However, even $16.5 \%$ of them admitted to having fear sometimes or always. Additionally, only $58.1 \%$ of participants would use voluntary dispensing error reporting system. Conclusion: Serbian community pharmacists are aware of the existing risk in medicines dispensing and the corrective actions identified should be put into practice so as to manage them prospectively. Although the results indicate good practice in incidents reporting, conducting tailored educations and building of safety culture is necessary to improve patient safety.
\end{abstract}

Key words: Dispensing errors, Community pharmacy, Patient safety, Pharmacists' attitudes, Risk management, Systems approach.

\section{INTRODUCTION}

In recent years, providing safe and highquality health care services has become an international priority. ${ }^{1}$ Ever since the consequences of medication errors have been described in landmark report "To Err Is Human", 2 this topic has been prioritized by World Health Organization (WHO) ${ }^{3}$ and numerous policy documents and research studies have been published highlighting growing concerns about the frequency and impact of medication errors. ${ }^{4-7}$

Dispensing of medicines traditionally represents one of the main pharmacists' activities in the provision of health care. ${ }^{8}$ However, this process is highly complex and consists of a number of steps that have to be performed before the medicine is issued to the patient. As errors can arise at each stage of this process and thus seriously affect patients' health care and their therapeutic outcomes, dispensing is one of the major concerns for the pharmaceutical profession. ${ }^{8}$
Submission Date: 17-12-2018; Revision Date: 15-03-2019; Accepted Date: 27-11-2019

DOI: 10.5530/ijper.54.1.23 Correspondence: Dr. Tatjana Stojkovic, Merck Sharp and Dohme Serbia Ltd, Omladinskih Brigada 90A, Belgrade11073, SERBIA.

Phone: +38164 3919728

E-mail: stojkovic.tatjana@ hotmail.com

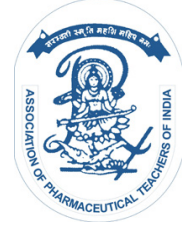

www.ijper.org 
Errors that may arise during the medicines dispensing process include "any deviation from the prescriber's order, made by staff in the pharmacy when distributing medications to nursing units or to patients in an ambulatory pharmacy setting". " Additionally, in accordance with the pharmacists' emerging role in providing patient-focused and individually tailored pharmaceutical care, failures to identify and correct prescribing faults and to minimize the risk of administration errors by appropriate patient counselling, have been recognized as significant additional categories of dispensing incidents. ${ }^{10}$ Fortunately, not all of these errors result in causing harm and the so-called near misses or close calls are defined as "an act of commission or omission that could have harmed the patient but did not do so as a result of chance, prevention or mitigation". ${ }^{11}$ However, it is of utmost importance to report this type of errors, since it represents significant step in preventing future adverse events and improving patient safety. ${ }^{12,13}$

Raising awareness of the risks related to the medicines dispensing has resulted in an increasing number of studies over the last decade. The results of international studies conducted in community pharmacies around the world indicate a wide range of dispensing error rates, which are reported to vary from $0.01 \%{ }^{14}$ to $24 \% .^{15}$ These findings indicate a need for further safety improvement, especially because pharmacies dispense such enormous quantities of medicines that even a low occurrence rate equals a substantial number of actual failures. ${ }^{16}$ Furthermore, safety-related concerns occurring in community pharmacies are identified as particularly relevant, because the vast majority of patients are habitually issued medicines at primary level of healthcare.

Pharmacists have a unique role in detection and prevention of medication errors occurring during the use of medicines and therefore, in improving the quality of pharmaceutical care services. ${ }^{17-19}$ Therefore, it is of crucial importance to examine their attitudes related to the risk management in the medicines dispensing process, as well as their practice in reporting of patient safety incidents incurred.

In recent years, several studies aiming to assess this topic have been performed in countries such as Australia, ${ }^{20}$ Ethiopia ${ }^{21}$ and Saudi Arabia. ${ }^{22}$ Here pharmacists have jointly expressed their belief that the risk of dispensing errors is increasing and have nominated various contributing factors as well as preventive actions perceived as important in reducing the risk of dispensing errors. However, despite the fact that the number of studies on medication errors arising in pharmacy practice has increased over the last decade, the problem of lack of research is still existent, particularly in developing countries such as Serbia. In this country, monitoring and reporting of the indicators on quality of healthcare, including those related to patient safety, has been performed as obligatory activity. When it comes to the pharmacy practice, the knowledge of dispensing errors is based largely on reporting by pharmacists. Namely, Institute of Public Health of Serbia has been continuously collecting and evaluating pharmacists' reports on incidents occurred during dispensing. However, types and causes of these incidents have neither been systematically analyzed, nor have corrective actions been developed and implemented, so far.

Therefore, general objective of this study was to explore the attitudes Serbian community pharmacists take towards medicine dispensing errors. More specifically, this study attempted to identify their attitudes regarding main factors leading to patient safety incidents in community pharmacies and potential interventions that could be made to prevent them, as well as to assess their practice in reporting of patient safety incidents incurred. This would be particularly significant bearing in mind the lack of research in this area in developing countries as well as at the primary level of pharmacy practice where the majority of patients are habitually issued medicines.

\section{MATERIALS AND METHODS}

\section{Study design}

A cross-sectional survey was performed by distributing a self-administered questionnaire to a nationwide, representative sample of community pharmacists in Serbia. The questionnaire was adopted from the study conducted by Peterson et al..$^{20}$ and slightly modified. After that, a draft version of the questionnaire was piloted on a convenience sample of 38 pharmacy practitioners so as to assess its readability, feasibility, question design and comprehension. Furthermore, the equivalence reliability of the questionnaire had been analyzed using Cronbach's alpha coefficient and the internal consistency for the overall instrument was found to be satisfactory $(\alpha=0.828)$.

Following the pilot study, some minor changes were made with regards to wording, formatting and questionnaire layout and the final version was distributed to the licensed community pharmacists from both stateand privately-owned pharmacies. The sampling frame included a list of community pharmacists registered as regular members of the Pharmaceutical Chamber of Serbia (PCS). This registration is obligatory for all pharmacists practicing in community pharmacies, in order 
to obtain licenses. The list comprised 6.096 pharmacists from both state- and privately-owned pharmacies and for the study power of $1-\beta=80 \%$ and statistical significance $\alpha=0.05$ the total sample size was calculated to be 361, according to the procedure defined by Krejcie and Morgan. ${ }^{23}$ This anticipated sample size $(n=361)$ was then adjusted in accordance with the potential drop-out rate $(\mathrm{d}=20 \%)$, using the formula $n=\mathrm{n} /(1-\mathrm{d} / 100)$, based on which the total number of required subject for participation in the study was $451 .{ }^{24}$

In order to involve pharmacists from all geographical areas in the research, the sample was further stratified by dividing the sampling frame into homogeneous subgroups (strata) based on official PCS classification system featuring 4 regional branches (PCS branch Beograd, Kragujevac, Vojvodina and Nis with Kosovo and Metohija listed 2.251, 1.456, 1.358 and 1.031 regular members, respectively). Accordingly, the sample size of each stratum was calculated using a proportionate stratification and equaled 167, 108, 100 and 76 for PCS branch Beograd, Kragujevac, Vojvodina and Nis with Kosovo and Metohija, respectively. Additionally, in order to obtain heterogeneous sample composition in terms of community pharmacies' types, participant recruitment plan included all state-owned pharmacies ${ }^{25}$ as well as all randomly selected privately-owned pharmacies (both having ${ }^{26}$ and not having a signed contract with the National Health Insurance Fund (NHIF) for dispensing prescription medicines from the Reimbursement list).

The study was conducted in accordance with the principles of the Declaration of Helsinki and approved by the Ethics Committee for Clinical Trials of the Faculty of Pharmacy, University of Belgrade, Serbia (Dossier No. 265/2).

\section{Questionnaire}

The questionnaire included 16 items classified into three main sections. The first section consisted of 6 items covering participants' characteristics, including sex, age, work experience, type of pharmacy practice site, dispensing hours per week and continuous dispensing hours per working day. The questions (1-6) were formatted in both multiple-choice and gapped sentences formats. The second section comprised 5 items addressing community pharmacists' attitudes towards dispensing errors, namely: (i) pharmacists' opinions on whether the risk and actual number of dispensing errors were increasing (questions 7 and 8), (ii) pharmacists' views on dispensing errors committed at their place of practice (question 10), as well as (iii) pharmacists' stance on the factors leading to dispensing errors occurrence (ques- tion 9) and potentially effective interventions to prevent them (question 11). Attitudes were explored using a three-point Likert scale (1="not significant", 2="partially significant", $3=$ =significant"). The third section consisted of 5 items, aiming to assess pharmacists' practice in reporting of dispensing errors, the existence of safety culture at their work place, as well as their opinions on the use of voluntary dispensing error reporting system (questions 12-16).

\section{Data collection and analysis}

The invitation for participation in the study was sent to all selected community pharmacies. After obtaining the approval for conducting the survey from the pharmacy director or owner, a defined number of the questionnaires, along with the cover letters, were distributed to the pharmacy practitioners by post or in person. Participation was completely voluntary, based on the information provided about the study aims and procedure, without offering any incentives. Informed consent was assumed by completion of the questionnaire, which was done anonymously and the participants were informed that the resulting findings would be aggregated and used in the scientific purposes only.

The data obtained from the completed questionnaires were entered, coded and analyzed using methods of descriptive and inferential statistics. Statistical analysis was performed using IBM SPSS Statistics for Windows (Version 21.0. Armonk, NY: IBM Corp.). Numerical data were reported as frequencies (percentage) for categorical variables and median, range and Interquartile Range (IQR) for continuous variables. The associations between variables were analyzed using Chi-square test for categorical variables, as the most appropriate for data presented in a form of Likert scale. The threshold of statistical significance was set to a conventional level at $p \leq 0.05$ for all analyses.

\section{RESULTS}

Out of 1,134 questionnaires distributed to community pharmacists, 1,004 completed returns were received thus accounting for a response rate of $88.5 \%$. Sociodemographic and pharmacy practice characteristics of the study participants are presented in Table 1. Most respondents were female ( $n=931,94.9 \%$ ), whereas their mean age was $40.9 \pm 9.9$ years (range: $21.0-65.0$ years) and mean work experience was $14.3 \pm 10.0$ years (range: $0.2-43.0$ years). The largest number of participants $(48.3 \%)$ performed medicines dispensing during 30-40 $\mathrm{h}$ per week, while the average time the respondents dis- 
pensed medications continuously was $3.6 \pm 2.0 \mathrm{~h}$ on a daily basis (range: $0.1-10.0$ ).

More than the third of the respondents ( $n=354,35.4 \%)$ indicated that the risk of dispensing errors was increasing. Similarly, about $21 \%(n=209)$ believed that the actual errors in dispensing were becoming more common in pharmacy practice.

Almost half the respondents $(n=487,49 \%)$ demonstrated the awareness of dispensing errors being committed at their workplace during the past 6 months, which were detected only after the patient had already left the pharmacy. The mean number of such occurrences was $2.3 \pm 2.4$ (range: $1-20$ ), while the total number of dispensing errors reported by all respondents in the timeframe spanning previous 6 months was 941 .

Pharmacists' attitudes towards the factors contributing to the occurrence of dispensing errors were explored using the three-point Likert scale and the results are presented in Table 2. The main causative factors identified included: illegible prescriber's handwriting (44.2\%), interruptions and distractions during medicines dispensing $(39.2 \%)$, as well as pharmacists' work overload $(37.8 \%)$ and understaffed shifts $(36.3 \%)$. Pharmacists' standpoints were further assessed with a view to identifying preventive measures against dispensing errors, by using the three-point Likert scale (Table 3). Various corrective actions were considered as important strategies for risk amelioration. The following ones were perceived as potentially the most effective: providing pharmacists with continuing education, particularly in relation to the newly registered drugs (71\%), reducing the avoidable interruptions during dispensing $(63.9 \%)$, increasing the number of pharmacy staff per shift $(61.9 \%)$, devoting more time to patients' counseling along with involving them more actively in the therapy management (60.3\%) and organizing pharmacists' training in the risk minimization strategies and interventions (57.8\%).

The Pearson Chi-square test was used to examine the association between the socio-demographic characteristics of the respondents and their attitudes regarding the factors contributing to the occurrence of dispensing errors, as well as the strategies for their reduction. The existence of differences in relation to the perceived significance of causative factors was observed between the defined groups within sex (1 factor), age (4 factors), work experience (6 factors) and type of community pharmacy ( 9 factors) (Table 4). Participants under 35 years of age, with $\leq 10$ years of work experience and working in privately-owned pharmacies stated in statistically significantly higher percentage that work overload $\left(\chi 2=14.698 . \mathrm{d}_{f}=2, p=0.001, \chi^{2}=22.056, \mathrm{~d}_{f}=2, p<0.001\right.$ and $\chi^{2}=30.957, \mathrm{~d}_{f}=2, p<0.001$, respectively), insuf- ficient time for patient counseling $(\chi 2=12.125, \mathrm{df}=2$, $p=0.002, \chi^{2}=11.089, \mathrm{~d}_{f}=2, p=0.04$ and $\chi^{2}=33.393$, $\mathrm{d}_{f}=2, p<0.001$, respectively) and interruptions and distractions during dispensing $\left(\chi 2=8.848, \mathrm{~d}_{f}=2, p=0.012\right.$, $\chi^{2}=6.823, \mathrm{~d}_{f}=2 p=0.033$ and $\chi^{2}=23.586, \mathrm{~d}_{f}=2, p<0.001$, respectively) were significant causative factors for the occurrence of errors. Additionally, pharmacists under 35 years of age and with $\leq 10$ years of work experience were more likely to consider illegible prescriber's handwriting as a factor of importance for the occurrence of errors $\left(\chi^{2}=7.649, \mathrm{~d}_{f}=2, p=0.022\right.$ and $\chi 2=9.947, \mathrm{~d}_{f}=2, p=0.007$, respectively), while participants with $\leq 10$ years of work experience and working in privately-owned pharmacies constituted a higher proportion when it comes to perceived significance of the understaffed shifts $\left(\chi 2=6.093, \mathrm{~d}_{f}=2, p=0.048\right.$ and $\chi^{2}=17.412, \mathrm{~d}_{f}=2, p<0.001$, respectively) and inadequate environmental conditions $\left(\chi 2=7.001, \mathrm{~d}_{f}=2, p=0.03\right.$ and $\chi^{2}=11.751, \mathrm{~d}_{f}=2, p=0.003$, respectively). Sex of the respondents and type of pharmacy significantly influenced their attitudes about the importance of job dissatisfaction, so male participants and pharmacists working in privately-owned pharmacies were more likely to opinion this factor as significant $\left(\chi 2=7.687, \mathrm{~d}_{f}=2, p=0.021\right.$ and $\chi^{2}=11.634, \mathrm{~d}_{f}=2, p=0.003$, respectively). The survey revealed additional differences between pharmacists' attitudes based on pharmacy ownership structure: substantially higher percentage of pharmacists practicing in state-owned pharmacies perceived sound-alike/ look-alike drug names as an important error causative factor $\left(\chi 2=7.262, \mathrm{~d}_{f}=2, p=0.026\right)$, while their colleagues from privately-owned pharmacies did this in relation to the high prescription volume and existence of generic brands $\left(\chi^{2}=11.106, \mathrm{~d}_{f}=2, p=0.004\right.$ and $\chi^{2}=8.481, \mathrm{~d}_{f}=2$, $p=0.014$, respectively).

When it comes to perceived significance of strategies for preventing errors in the medicines dispensing process, the existence of differences was observed between the defined groups within sex (4 factors), age (3 factors), work experience (6 factors) and type of community pharmacy (4 factors) (Table 5). Female pharmacists, under 35 years of age, with $\leq 10$ years of work experience and working in privately-owned pharmacies, constituted a higher proportion of participants who stated that reducing the pharmacists' workload was significant measure for preventing dispensing errors occurrence $\left(\chi^{2}=10.885, \mathrm{df}=2, p=0.004, \chi^{2}=8.078, d_{f}=2, p=0.018\right.$, $\chi^{2}=8.304, \mathrm{df}=2, \quad p=0.016$ and $\chi^{2}=25.382, \mathrm{~d}_{f}=2$, $p=0.001$, respectively). Additionally, female pharmacists were more likely to opinion increasing the number of pharmacy staff per shift $\left(\chi 2=8.605, \mathrm{~d}_{f}=2, p=0.014\right)$, providing pharmacists with continuing education, par- 
ticularly regarding newly registered drugs $(\chi 2=17.072$, $\left.\mathrm{d}_{f}=2, p<0.001\right)$, as well as organizing pharmacists' training related to the risk management $\left(\chi^{2}=9.966, \mathrm{~d}_{f}=2\right.$, $p=0.007)$ as significant risk minimization strategies for the medicines dispensing process. Similarly, pharmacists working in privately-owned pharmacies did this in relation to the increasing the number of pharmacy staff per shift $\left(\chi^{2}=6.749, \mathrm{~d}_{f}=2, p=0.034\right)$ as well as implementation of control mechanisms for checking the dispensing procedure $\left(\chi^{2}=6.147, d_{f}=2, p=0.046\right)$. When it comes to defining an operative dispensing workflow, the differences between the groups within age, work experience and type of community pharmacy have been observed, so pharmacists over 36 years, with $\geq 11$ years of work experience and working in state-owned pharmacies expressed in a statistically significantly higher percentage a positive attitude towards the potential impact of this corrective measure $\left(\chi 2=6.716, d_{f}=2, p=0.035, \chi^{2}=8.929\right.$, $d_{f}=2, p=0.012$ and $\chi^{2}=14.795, d_{f}=2, p=0.001$, respectively). On the other hand, their younger colleagues (i.e. pharmacists under 35 years and with $\leq 10$ years of work experience) were more prone to accentuate the significance of separate storage of sound-alike/lookalike drugs $\left(\chi^{2}=19.211, d_{f}=2, p<0.001\right.$ and $\chi^{2}=13.561$, $d_{f}=2, p=0.001$, respectively). The work experience also influenced the attitudes towards improving the packaging and labeling of drugs, where pharmacists with $\geq 11$ years of work experience were more likely to consider this strategy as significant $\left(\chi 2=6.776, d_{f}=2, \mathrm{p}=0.034\right)$, while substantially higher proportion of respondents with $\leq 10$ years of work experience acknowledged the importance of electronic transmission of prescriptions to the pharmacy $\left(\chi 2=6.203, d_{f}=2, p=0.045\right)$ and implementation of control mechanisms for checking the dispensing procedure $\left(\chi 2=12.473, \mathrm{~d}_{f}=2, p=0.002\right)$.

In relation to the pharmacists' practice in dispensing errors reporting, the majority $(n=833,85.2 \%)$ of respondents stated that they had routinely reported dispensing incidents occurred, where the participants under 35 years of age, with $\leq 10$ years of work experience service and working in privately-owned pharmacies constituted a significantly higher proportion of all respondents who had reported dispensing errors $\left(\chi 2=6.287, d_{f}=2\right.$, $p=0.043, \chi^{2}=9.735, d_{f}=2, p=0.008$ and $\chi^{2}=11.899$, $d_{f}=2, \mathrm{p}=0.003$, respectively) (Table 6 ). The analysis further revealed that the largest number of pharmacists $(n=900,91.5 \%)$ indicated that discussions about these cases were conducted openly at their place of practice, where the participants working in privately-owned pharmacies were more likely to do so $\left(\chi 2=23.173, \mathrm{~d}_{f}=2\right.$, $p<0.001)$. Although most $(n=818,83.6 \%)$ of the participants declared having no fear about being blamed and $\backslash$ or sanctioned by their superiors should they decide to report dispensing errors, as many as $16.5 \%(n=161)$ of them admitted to having such fear sometimes or always. The opinions were also sought on whether reporting any type of dispensing errors, regardless of their severity, was deemed to be important and the large proportion of practitioners $(n=860,87.4 \%)$ demonstrated positive attitude towards this issue. However, when asked whether they would use voluntary dispensing error reporting system, if established, only 58.1\% $(\mathrm{N}=561)$ of them responded positively, while $41.9 \%$ $(n=404)$ said they would use it sometimes or never. Additionally, it was found that the respondents under 35 years of age, with $\leq 10$ years of work experience and practicing in privately-owned pharmacies were more likely to use voluntary dispensing error reporting system $\left(\chi 2=36.389, d_{f}=2, p<0.001, \chi 2=46.035, d_{f}=2, p<0.001\right.$ and $\chi^{2}=41.398, d_{f}=2, p<0.001$, respectively) (Table 6).

\section{DISCUSSION}

Along with the development of modern approach to medication errors, an increasing emphasis has been laid

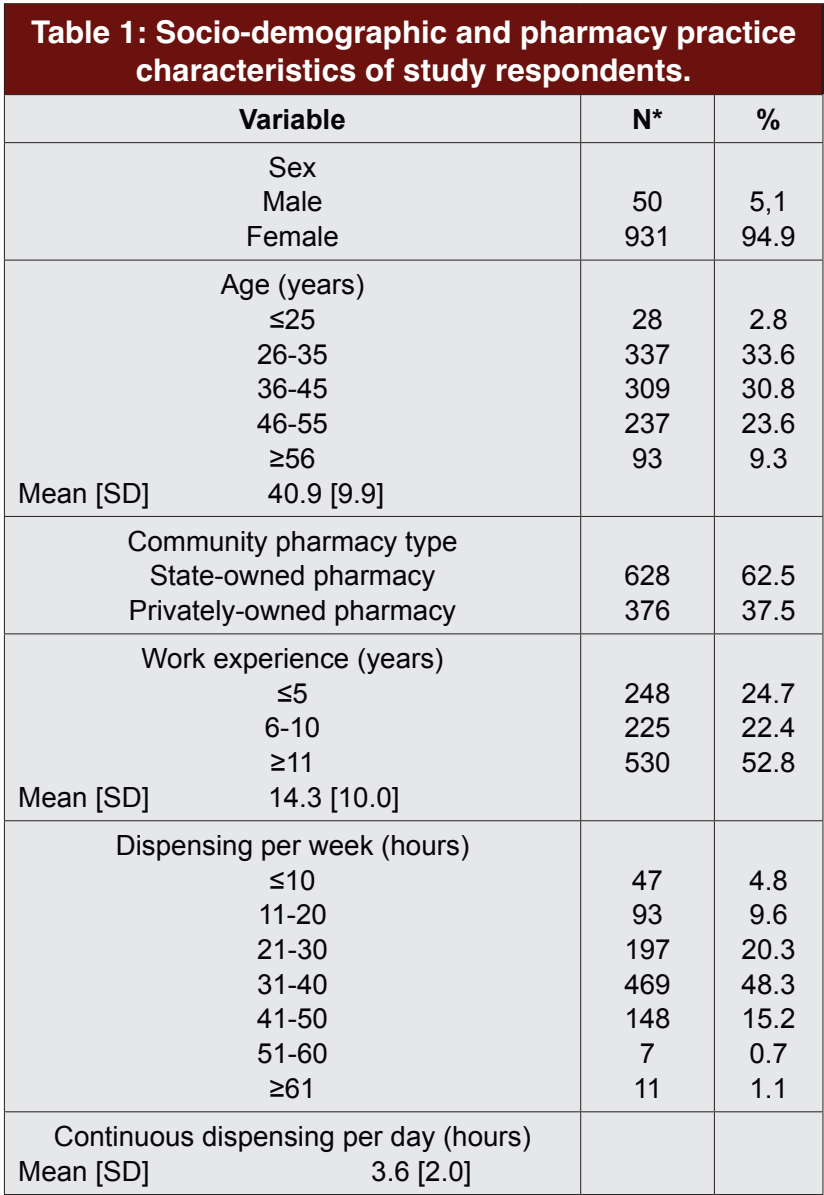

Note. $S D=$ Standard Deviation; *Responses may total less than 1004 as not all respondents answered every question; 


\begin{tabular}{|c|c|c|c|}
\hline \multirow[t]{2}{*}{ Variable } & \multicolumn{3}{|c|}{ Response } \\
\hline & $\begin{array}{c}\text { Significant } \\
\text { contributing factor } \\
\mathrm{N}(\%)\end{array}$ & $\begin{array}{c}\text { Partially significant } \\
\text { factor } \\
\text { N (\%) }\end{array}$ & $\begin{array}{c}\text { Not a significant } \\
\text { contributing factor } \\
\text { N (\%) }\end{array}$ \\
\hline Illegible prescriber's handwriting & $435(44.2 \%)$ & $464(47.3 \%)$ & $83(8.5 \%)$ \\
\hline Sound-alike/look-alike drug names & $137(14.2 \%)$ & $589(61 \%)$ & $240(24.8 \%)$ \\
\hline Existence of generic brands & $45(4.7 \%)$ & $302(31.6 \%)$ & $609(63.7 \%)$ \\
\hline Drug packaging and labeling & $127(13.3 \%)$ & $466(48.8 \%)$ & $362(37.9 \%)$ \\
\hline Pharmacist's work overload & $368(37.8 \%)$ & $497(51.1 \%)$ & $108(11.1 \%)$ \\
\hline Job dissatisfaction & $147(15.4 \%)$ & $446(46.6 \%)$ & $364(38 \%)$ \\
\hline High prescription volume & $237(24.8 \%)$ & $525(54.9 \%)$ & $194(20.3 \%)$ \\
\hline Understaffed shifts & $350(36.3 \%)$ & $484(50.2 \%)$ & $131(13.5 \%)$ \\
\hline $\begin{array}{l}\text { Inadequate environmental conditions (poor lightning, } \\
\text { noise, cluttered work space etc.) }\end{array}$ & $242(25.1 \%)$ & $493(51.1 \%)$ & $229(23.8 \%)$ \\
\hline Interruptions and distractions during dispensing & $383(39.2 \%)$ & $515(52.7 \%)$ & $79(8.1 \%)$ \\
\hline Insufficient time for patient counseling & $291(29.9 \%)$ & $531(54.5 \%)$ & $152(15.6 \%)$ \\
\hline
\end{tabular}

\begin{tabular}{|c|c|c|c|}
\hline \multirow[t]{2}{*}{ Variable } & \multicolumn{3}{|c|}{ Response } \\
\hline & $\begin{array}{l}\text { Significant } \\
\text { N (\%) }\end{array}$ & $\begin{array}{c}\text { Partially } \\
\text { significant } \\
\mathrm{N}(\%)\end{array}$ & $\begin{array}{c}\text { Not } \\
\text { significant } \\
\text { N (\%) }\end{array}$ \\
\hline Electronic transmission of prescriptions to the pharmacy & $420(44 \%)$ & $396(41.5 \%)$ & $139(14.6 \%)$ \\
\hline Separate storage of sound-alike/look-alike drugs & $481(49.9 \%)$ & $421(43.7 \%)$ & $62(6.4 \%)$ \\
\hline Improving the packaging and labeling of drugs & $458(47.5 \%)$ & $434(45 \%)$ & $73(7.6 \%)$ \\
\hline Defining an operative dispensing workflow & $425(44.3 \%)$ & $410(42.8 \%)$ & $124(12.9 \%)$ \\
\hline Implementation of control mechanisms for checking the dispensing procedure & $548(56.8 \%)$ & $359(37.2 \%)$ & $58(6 \%)$ \\
\hline Reducing the pharmacists' workload & $553(56.4 \%)$ & $383(39 \%)$ & $45(4.6 \%)$ \\
\hline Increasing the number of pharmacy staff per shift & $605(61.9 \%)$ & $327(33.5 \%)$ & $45(4.6 \%)$ \\
\hline $\begin{array}{c}\text { Providing pharmacists with continuing education, particularly regarding newly } \\
\text { registered drugs }\end{array}$ & $689(71 \%)$ & $253(26.1 \%)$ & $29(3 \%)$ \\
\hline Reducing the avoidable interruptions during dispensing & $621(63.9 \%)$ & $318(32.7 \%)$ & $33(3.4 \%)$ \\
\hline Devoting more time to patients' counseling, along with involving them more actively & $586(60.3 \%)$ & $346(35.6 \%)$ & $40(4.1 \%)$ \\
\hline $\begin{array}{l}\text { Organizing pharmacists' training related to the risk minimization strategies and } \\
\text { interventions }\end{array}$ & $561(57.8 \%)$ & $360(37.1 \%)$ & $50(5.1 \%)$ \\
\hline
\end{tabular}

on the prospective risk analysis, since this type of risk management allows implementation of corrective measures before a preventable adverse event has occurred and caused harm to the patient. ${ }^{27}$ It is exactly the reason why it is of crucial importance to examine pharmacists' attitudes related to the risk management in the medicines dispensing process, in order to identify causes of dispensing errors as well as potential preventive measures to avoid them in the community pharmacy setting. Study results indicate that more than a third of the participants in this study (35.4\%) indicated that the risk of dispensing errors was increasing, which was lower compared to $44.7 \%, 62 \%$ and $82.2 \%$ of the community pharmacists from Ethiopia, ${ }^{24}$ Saudi Arabia ${ }^{25}$ and Australia, ${ }^{18}$ respectively, who demonstrated such attitude. Additionally, almost half the respondents (49\%) demonstrated the awareness of dispensing errors being committed at their workplace during the past 6 months, whereby the total number reported by all respondents was 941 . Although these rate was lower than those reported in a study performed in Australia ${ }^{18}$ where $70.9 \%(n=134)$ of pharmacists expressed awareness of dispensing errors that had left the pharmacy undetected in the past 6 months, with their total number of 498 , the results obtained still indicated a significant self-reported rate of errors and the necessity of prospective re-design of dispensing process in Serbian community pharmacies, in order to prevent such occurrences. 
Table 4: Association between the respondents' socio-demographic characteristics and their attitudes regarding the factors contributing to the occurrence of dispensing errors.

\begin{tabular}{|c|c|c|c|c|}
\hline \multirow{2}{*}{ Factors for dispensing errors } & \multicolumn{3}{|c|}{ Independent variables } \\
\cline { 2 - 5 } & Sex & Age & $\begin{array}{c}\text { Work } \\
\text { experience }\end{array}$ & $\begin{array}{c}\text { Community } \\
\text { pharmacy type }\end{array}$ \\
\hline Illegible prescriber's handwriting & $p=0.409$ & $p=0.022^{\S}$ & $p=0.007^{\S}$ & $p=0.396$ \\
\hline Sound-alike/look-alike drug names & $p=0.795$ & $p=0.890$ & $p=0.712$ & $p=0.026^{\S}$ \\
\hline Existence of generic brands & $p=0.578$ & $p=0.256$ & $p=0.694$ & $p=0.014^{\S}$ \\
\hline Drug packaging and labeling & $p=0.12$ & $p=0.207$ & $p=0.346$ & $p=0.454$ \\
\hline Pharmacist's work overload & $p=0.451$ & $p=0.001^{\S}$ & $p<0.001^{\S}$ & $p<0.001^{\S}$ \\
\hline Job dissatisfaction & $p=0.021^{\S}$ & $p=0.390$ & $p=0.119$ & $p=0.003^{\S}$ \\
\hline High prescription volume & $p=0.59$ & $p=0.269$ & $p=0.296$ & $p=0.004^{\S}$ \\
\hline Understaffed shifts & $p=0.272$ & $p=0.089$ & $p=0.048^{\S}$ & $p<0.001^{\S}$ \\
\hline Inadequate environmental conditions (poor lightning, noise, & $p=0.251$ & $p=0.098$ & $p=0.03^{\S}$ & $p=0.003^{\S}$ \\
\hline cluttered work space etc.) & $p=0.582$ & $p=0.012^{\S}$ & $p=0.033^{\S}$ & $p<0.001^{\S}$ \\
\hline Interruptions and distractions during dispensing & $p=0.446$ & $p=0.002^{\S}$ & $p=0.04^{\S}$ & $p<0.001^{\S}$ \\
\hline Insufficient time for patient counseling & & & & \\
\hline
\end{tabular}

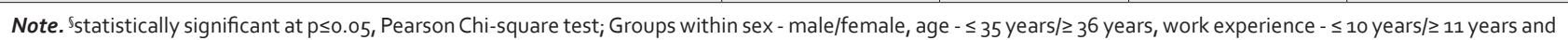
type of community pharmacy - state-owned/privately-owned pharmacy;

\begin{tabular}{|c|c|c|c|c|}
\hline \multirow[t]{2}{*}{ Interventions for dispensing errors minimization } & \multicolumn{4}{|c|}{ Independent variables } \\
\hline & Sex & Age & $\begin{array}{c}\text { Work } \\
\text { experience }\end{array}$ & $\begin{array}{l}\text { Community } \\
\text { pharmacy type }\end{array}$ \\
\hline Electronic transmission of prescriptions to the pharmacy & $p=0.159$ & $p=0.051$ & $p=0.045^{\S}$ & $p=0.344$ \\
\hline Separate storage of sound-alike/look-alike drugs & $p=0.949$ & $p<0.001^{\S}$ & $p=0.001^{\S}$ & $p=0.259$ \\
\hline Improving the packaging and labeling of drugs & $p=0.057$ & $p=0.540$ & $p=0.034^{\S}$ & $p=0.618$ \\
\hline Defining an operative dispensing workflow & $p=0.208$ & $p=0.035^{\S}$ & $p=0.012^{\S}$ & $p=0.001^{\S}$ \\
\hline $\begin{array}{l}\text { Implementation of control mechanisms for checking the dispensing } \\
\text { procedure }\end{array}$ & $p=0.787$ & $p=0.174$ & $p=0.002^{\S}$ & $p=0.046^{\S}$ \\
\hline Reducing the pharmacists' workload & $p=0.004 \S$ & $p=0.018^{\S}$ & $p=0.016 \S$ & $p<0.001^{\S}$ \\
\hline Increasing the number of pharmacy staff per shift & $p=0.014^{\S}$ & $p=0.365$ & $p=0.249$ & $p=0.034 \S$ \\
\hline $\begin{array}{l}\text { Providing pharmacists with continuing education, particularly regarding } \\
\text { newly registered drugs }\end{array}$ & $p<0.001^{\S}$ & $p=0.707$ & $p=0.854$ & $p=0.363$ \\
\hline Reducing the avoidable interruptions during dispensing & $p=0.113$ & $p=0.724$ & $p=0.756$ & $p=0.849$ \\
\hline $\begin{array}{l}\text { Devoting more time to patients' counseling, along with involving them more } \\
\text { actively }\end{array}$ & $p=0.224$ & $p=0.737$ & $p=0.467$ & $p=0.648$ \\
\hline $\begin{array}{l}\text { Organizing pharmacists' training related to the risk minimization strategies } \\
\text { and interventions }\end{array}$ & $p=0.007^{\S}$ & $p=0.355$ & $p=0.134$ & $p=0.485$ \\
\hline
\end{tabular}

Note. Sstatistically significant at $p \leq 0.05$, Pearson Chi-square test; Groups within sex - male/female, age - $\leq 35$ years/ $\geq 36$ years, work experience - $\leq 10$ years/ $\geq 11$ years and type of community pharmacy - state-owned/privately-owned pharmacy;

When it comes to the factors contributing to the occurrence of errors in the medicines dispensing process, illegible prescriber's handwriting (44.3\%) was perceived as the most significant one. This is in line with the causes of dispensing errors which were prospectively identified in the Failure Mode and Effects Analysis (FMEA) study carried out in the Serbian community pharmacy setting, ${ }^{10}$ as well as with the results of other studies which examined pharmacists' attitudes on this issue. ${ }^{20-22}$ In this case, the implementation of electronic prescribing systems, integrated with pharmacies could be efficient strategy for reducing possible selection and transcription errors during the dispensing process, which is in line with other studies, where the effectiveness of this intervention for improving patient safety and reducing error rates in pharmacy practice has been confirmed. ${ }^{28}$ 
Table 6: Association between the respondents' socio-demographic characteristics and their practice of dispensing errors reporting.

\begin{tabular}{|c|c|c|c|c|}
\hline & \multicolumn{4}{|c|}{ Independent variables } \\
\hline & Sex & Age & $\begin{array}{c}\text { Work } \\
\text { experience }\end{array}$ & $\begin{array}{l}\text { Community } \\
\text { pharmacy type }\end{array}$ \\
\hline Reporting dispensing errors incurred & $p=0.593$ & $p=0.043^{\S}$ & $p=0.008^{\S}$ & $p=0.003^{\S}$ \\
\hline $\begin{array}{l}\text { Having fear of being blamed and punished by superiors when reporting } \\
\text { dispensing errors }\end{array}$ & $p=0.473$ & $p=0.755$ & $p=0.699$ & $p=0.062$ \\
\hline Discussing about dispensing errors incurred openly at the place of practice & $p=0.302$ & $p=0.300$ & $p=0.424$ & $p<0.001^{\S}$ \\
\hline $\begin{array}{l}\text { Willingness to report any type of dispensing errors, regardless of their } \\
\text { severity }\end{array}$ & $p=0.317$ & $p=0.190$ & $p=0.358$ & $p=0.893$ \\
\hline $\begin{array}{l}\text { Willingness to use the voluntary dispensing error reporting system, if } \\
\text { established }\end{array}$ & $p=0.586$ & $p<0.001^{\S}$ & $p<0.001^{\S}$ & $p<0.001^{\S}$ \\
\hline
\end{tabular}

Note. Sstatistically significant at $p \leq 0.05$, Pearson Chi-square test; Groups within sex - male/female, age $-\leq 35$ years/ $\geq 36$ years, work experience $-\leq 10$ years/ $\geq 11$ years and type of community pharmacy - state-owned/privately-owned pharmacy;

Furthermore, one of the top critical points of concern identified included pharmacists' work overload and understaffed shifts. Here a useful measure would include service structuring, that is, defining a maximum safe dispensing workload per pharmacist, which has already been established in some countries. For example, in Australia, the Pharmacy Board of New South Wales has issued a recommendation that a pharmacist should not process more than 12-15 prescriptions per hour, in order to reduce the probability of error occurrence, which is in line with the views expressed by the pharmacists in the study conducted by Peterson et al. ${ }^{20}$ in relation to the maximum safe dispensing load, which amounted to approximately 150 prescriptions per pharmacist per day, that is, 17 prescriptions per hour. Furthermore, a strategy for proactive action on these types of systemic causes of errors includes defining the number of employees according to workload, i.e. the number of prescriptions processed daily at the pharmacy. For example, the Pharmacy Board of Australia has recommended that the number of employed pharmacists should be increased if the number of prescriptions processed per pharmacist per day exceeds 200, in order to provide them with enough time for adequate prescription processing and medicines dispensing in accordance with standards and guidelines. ${ }^{29}$

Additionally, the provision of pharmacists with continuing training in pharmacotherapy is proposed as one of the key measures for improving the safety of medicines dispensing processes. This educational intervention has proved to be highly effective for the advancement of pharmaceutical care. ${ }^{30,31}$

Furthermore, one of the main corrective actions included devoting more time to patients' counseling, along with involving them more actively. Over the last decade, the importance of abandoning the paternalis- tic approach in communication with patients and moving to the shared decision-making has been increasingly emphasized. In accordance with the introduction of the above-mentioned patient-centered approach as a "gold standard" in the provision of health care, ${ }^{32}$ the relationship between pharmacists and patients has also been changing, whereby the patient becomes an active partner in communication, with whom it is necessary to establish a professional relationship based on trust, open communication and joint decision-making. ${ }^{33}$

In addition, the analysis of survey results revealed that the pharmacists' age, work experience and type of community pharmacy influenced significantly their perception of the contribution of the above-mentioned factors to the occurrence of errors, while sex has not demonstrated significant impact. Interruptions and distractions during dispensing, work overload and insufficient time for patient counseling were more frequently regarded as important causative factors for dispensing errors among participants under 35 years of age, with $\leq 10$ years of work experience and practicing in privately-owned pharmacy. Similar results were obtained in the study carried out by Peterson et al. ${ }^{20}$ where it was found that the increase in work experience statistically significantly correlated with the decline in the perceived importance of the illegible prescriber's handwriting $(p<0.05)$ and insufficient time for patient counseling $(p<0.05)$ as causative factors. Such results indicate that older pharmacists, with longer work experience and working in state-owned pharmacies have greater ability to detect and correct errors occurring in the medicines dispensing process, despite the presence of the aforementioned systemic factors. Accordingly, further proactive actions on the identified causes of errors as well as tailored educations and training in risk management are necessary, primarily directed at younger pharmacists with shorter 
work experience and working in privately-owned pharmacies.

Pharmacists who participated in this study acknowledge several preventive actions as important strategies for minimizing the risk of dispensing errors. In addition, the results obtained indicate that certain characteristics of Serbian community pharmacists, such as age, work experience and practicing site type, influenced significantly their perception of the effectiveness of corrective actions proposed in proactive risk reduction, while sex has not demonstrated significant impact. In accordance with this, the type of community pharmacy, as well as age and work experience of pharmacy staff should be taken into consideration during the prioritization of corrective measures for implementation in a particular community pharmacy.

Reporting of incidents occurred in community pharmacies by using standardized forms represents one of the main mechanisms for learning from the committed mistakes. However, a common practice of under-reporting of adverse events can be observed in this type of health care facilities. ${ }^{34-36}$ For instance, the results of the study carried out by Varadarajan et al. ${ }^{34}$ which aimed to compare direct observation and incident reporting as two methods for detecting dispensing errors, indicated that the number of errors identified by direct observation was 16 times higher than the number of those reported. Therefore, it is of critical importance to examine the pharmacists' practice in incident reporting, as well as their attitudes on this issue, in order to proactively address the detected barriers. The fact that majority $(85.2 \%)$ of respondents stated that they had routinely reported dispensing incidents and adverse events incurred, indicate good community pharmacists' practice in reporting of dispensing errors. However, it is necessary to further examine the current pharmacists' practice in dispensing errors reporting by using the direct observation method, in order to achieve an objective confirmation of the results obtained. Additionally, when asked about having fear of being blamed and $\backslash$ or sanctioned by their superiors should they decide to report dispensing errors, as many as $16.5 \%$ of participants admitted to having such fear sometimes or always? One of the main reasons for the fear of health care providers to report incidents incurred, includes the existence of the blame culture, which is considered to be highly present in community pharmacies. ${ }^{37}$ It is exactly the change in the organization's culture which represents the first step in raising awareness on the significance of incident reporting, from the one that is oriented towards blaming and punishing individuals for making a mistake, to the one that is focused on improving the system and the compe- tencies of employees, as well as on their encouragement to discuss the errors openly. ${ }^{38}$ Standardization of error detection system, simplification of reporting procedures and establishment of non-punitive environment may contribute significantly to reducing the risk related to dispensing errors and repetition of mistakes. Our results indicate the need for further building of patient safety culture in Serbian community pharmacies. This could be obtained by adaptation of safety culture assessment tools used for self-assessment of safety culture maturity level, such as the Manchester Patient Safety Assessment Framework (MaPSAF) ${ }^{39}$ or the Medication Safety Selfassessment (MSSA) for the Community/Ambulatory Pharmacy. ${ }^{40}$ Another potential way to achieve this is to establish a centralized, national voluntary reporting system for errors in the medicines dispensing, which would be anonymous. This type of reporting would enable pharmacy staff to analyze medication incidents and learn about their potential causes, which could lead to the change in their practice and consequently to the prevention of recurrence of these incidents in the future. National voluntary incident reporting systems have already been developed in a number of countries, such as Australia, the United States, Denmark, Canada, the Netherlands and England and Wales. ${ }^{36}$ However, only $58.1 \%$ of community pharmacists in Serbian study stated that they would use voluntary dispensing error reporting system, if established, whereby the respondents under 35 years of age, with $\leq 10$ years of work experience and practicing in privately-owned pharmacies were more likely to do so. Accordingly, it is necessary to conduct tailored educations with the aim of raising awareness of Serbian community pharmacists regarding the significance of medication errors and their reporting through this type of system, which should be primarily directed towards older pharmacists with longer work experience and working in state-owned pharmacies.

The strengths of this study include obtaining a national sample of pharmacists which is representative in terms of gender, type of community pharmacy and geographical areas. This is first study examining attitudes of Serbian pharmacists on the topic of dispensing errors, as well as evaluating number of self-reported dispensing incidents occurring at the primary level of pharmacy practice. However, certain limitations of this study include the lack of further collection of data on the number of items dispensed as well as on whether the harm occurred as a result of these self-reported errors. Additionally, linkage to the officially available quality indicators such as number of prescriptions processed per pharmacist was not possible due to difference in the included time frame. Accordingly, further research plans 
should include performing study on the incurred dispensing errors which would enable linkage to the quality indicators and thus evaluation of a percentage of errors as well as differentiation between near missed and cases where the error reached the patient. Additionally, recommendations to policy makers and practitioners include developing standardized quality management system in community pharmacies, service structuring, that is, defining a maximum safe dispensing workload per pharmacist, as well as conducting tailored educations on dispensing errors in order to raise the importance on their reporting. On the macro level, national legislation should be advanced in relation to the obligatory quality indicators which should be monitored.

Considering the lack of research in this area in developing countries, the study results provide additional insight in major factors contributing to the occurrence of errors, main remedial measures which could be effective in patient safety risk reduction, as well as pharmacists' practice in (non)reporting of dispensing incidents, its limitations and areas for improvement. Additionally, such study has not been performed in Europe, so it also provides opportunity to compare pharmacists' attitudes on this topic worldwide.

\section{CONCLUSION}

The results of the study on Serbian community pharmacists' attitudes towards risk management in the medicines dispensing suggest that they are aware of the existing risk related to this process, as well as the factual errors occurrence. Additionally, the main causative factors and corrective actions have been identified, which should be further put into practice in order to manage dispensing risks prospectively and prevent the occurrence of adverse events before causing harm to the patient. Finally, although the results obtained indicate good Serbian community pharmacists' practice in reporting of dispensing errors, further building of patient safety culture in community pharmacies as well as conducting tailored educations regarding medication errors is necessary, in order to improve patient safety and pharmaceutical service quality.

\section{ACKNOWLEDGEMENT}

The authors sincerely thank to all community pharmacists who participated in the study.

\section{CONFLICT OF INTEREST}

The authors declare no conflict of interest.

\section{ABBREVIATIONS}

FMEA: Failure Mode and Effects Analysis; IOM: Institute of Medicine; IQR: Interquartile range; NHIF: National Health Insurance Fund; PCS: Pharmaceutical Chamber of Serbia; WHO: World Health Organization.

\section{REFERENCES}

1. Stojkovic T, Marinkovic V, Krajnovic D, Tasic LJ, Milosevic-Georgiev A. Patient safety and medication errors in the provision of health care serviceschallenges for contemporary practice. Acta Medica Medianae. 2016;55(2):5764.

2. Donaldson MS, Corrigan JM, Kohn LT. To Err is Human: Building a Safer Health System. Washington, DC: National Academies Press. 2000.

3. World Health Organization. WHO Global Patient Safety Challenge: Medication Without Harm. World Health Organization: Geneva. 2017. [cited 2018 November 10]. Available from: https://apps.who. int/iris/bitstream/handle/10665/255263/WHO-HIS-SDS-2017.6-eng. pdf;jessionid=7FF7813F7F04B3C69AEE57E4A6294F44? sequence=1.

4. Department of Health. Building a Safer NHS for Patients: Implementing an Organisation with a Memory. London: Department of Health. 2002. [cited 2019 March 23]. Available from: http://158.132.155.107/posh97/private/GSP/ NHS.pdf.

5. Assiri GA, Shebl NA, Mahmoud MA, Aloudah N, Grant E, Aljadhey H, et al. What is the epidemiology of medication errors, error-related adverse events and risk factors for errors in adults managed in community care contexts? A systematic review of the international literature. BMJ Open. 2018;8(5):e019101.

6. Naples JG, Hanlon JT, Schmader KE, Semla TP. Recent literature on medication errors and adverse drug events in older adults. J Am Geriatr Soc. 2016;64(2):401-8.

7. Krzyzaniak N, Bajorek B. Medication safety in neonatal care: A review of medication errors among neonates. Ther Adv Drug Saf. 2016;7(3):102-19.

8. James KL, Barlow D, McArtney R, Hiom S, Roberts D, Whittlesea C. Incidence, type and causes of dispensing errors: A review of the literature. Int J Pharm Pract. 2009;17(1):9-30.

9. Cohen MR, Smetzer JL, Tuohy NR, Kilo CM. High-alert medications: Safeguarding against errors. Medication Errors. Washington, DC: American Pharmaceutical Association. 2007;317-411.

10. Stojkovic T, Marinkovic V, Jaehde U, Manser T. Using Failure mode and Effects Analysis to reduce patient safety risks related to the dispensing process in the community pharmacy setting. Res Social Adm Pharm. 2017;13(6):1159-66.

11. Erickson SM, Wolcott J, Corrigan JM, Aspden P. Patient safety: Achieving a new standard for care. Washington, DC: National Academies Press. 2003.

12. Kang HJ, Park H, Oh JM, Lee EK. Perception of reporting medication errors including near-misses among Korean hospital pharmacists. Medicine. 2017;96(39):e7795.

13. Sheikhtaheri A. Near misses and their importance for improving patient safety. Iran J Public Health. 2014;43(6):853-4.

14. Knudsen P, Herborg H, Mortensen AR, Knudsen M, Hellebek A. Preventing medication errors in community pharmacy: Frequency and seriousness of medication errors. Qual Saf Health Care. 2007;16(4):291-6.

15. Allan EL, Barker KN, Malloy MJ, Heller WM. Dispensing errors and counseling in community practice. Am Pharm. 1995;NS35(12):25-33.

16. Flynn EA, Barker KN, Carnahan BJ. National observational study of prescription dispensing accuracy and safety in 50 pharmacies. J Am Pharm Assoc. 2003;43(2):191-200.

17. Ashcroft D, Morecroft C, Parker D, Noyce P. Patient safety in community pharmacy: Understanding errors and managing risk. London: Royal Pharmaceutical Society of Great Britain. 2005.

18. Shah A. Pharmacy intervention in the medication-use process: The role of pharmacists in improving patient safety. The Hague (Netherlands): International Pharmaceutical Federation. 2009. [cited 2019 March 23]. Available from: https://www.fip.org/files/content/priority-areas/patient-safety/ patientsafetyadvidshah.pdf. 
19. Mangino PD. Role of the pharmacist in reducing medication errors. J Surg Oncol. 2004;88(3):189-94.

20. Peterson GM, Wu MS, Bergin JK. Pharmacists' attitudes towards dispensing errors: Their causes and prevention. J Clin Pharm Ther. 1999;24(1):57-71.

21. Asmelashe GD, Binega MG. Perception of Community Pharmacists towards Dispensing Errors in Community Pharmacy Setting in Gondar Town, Northwest Ethiopia. Biomed Res Int. 2017;2017:2137981.

22. Al-Arifi MN. Community pharmacists' attitudes toward dispensing errors at community pharmacy setting in Central Saudi Arabia. Saudi Pharm J. 2014;22(3):195-202.

23. Krejcie RV, Morgan DW. Determining sample size for research activities. Educ Psychol Meas. 1970;30(3):607-10.

24. Sakpal T. Sample size estimation in clinical trial. Perspect Clin Res. 2010;1(2):67.

25. Decret on the Healthcare Facilities Network Plan. "Official Gazette of the Republic of Serbia”, No. 42/2006, 119/2007, 84/2008, 71/2009, 85/2009, 24/2010, 6/2012, 37/2012, 8/2014, 92/2015, 111/2017, 114/2017, 13/2018 and $15 / 2018$.

26. National Health Insurance Fund. List of privately-owned community pharmacies and their branches with which HIF concluded contracts. 2016.

27. Stojkovic T, Marinkovic V, Manser T. Using Prospective Risk Analysis Tools to Improve Safety in Pharmacy Settings: A Systematic Review and Critical Appraisal. J Patient Saf. 2017.

28. Moniz TT, Seger AC, Keohane CA, Seger DL, Bates DW, Rothschild JM. Addition of electronic prescription transmission to computerized prescriber order entry: Effect on dispensing errors in community pharmacies. Am J Health Syst Pharm. 2011;68(2):158-63.

29. Pharmacy Board of Australia. Guidelines for dispensing of medicines. 2015;17. [cited 2019 March 23]. Available from: http://apps.who.int/medicinedocs/ documents/s17807en/s17807en.pdf.

\section{PICTORIAL ABSTRACT}

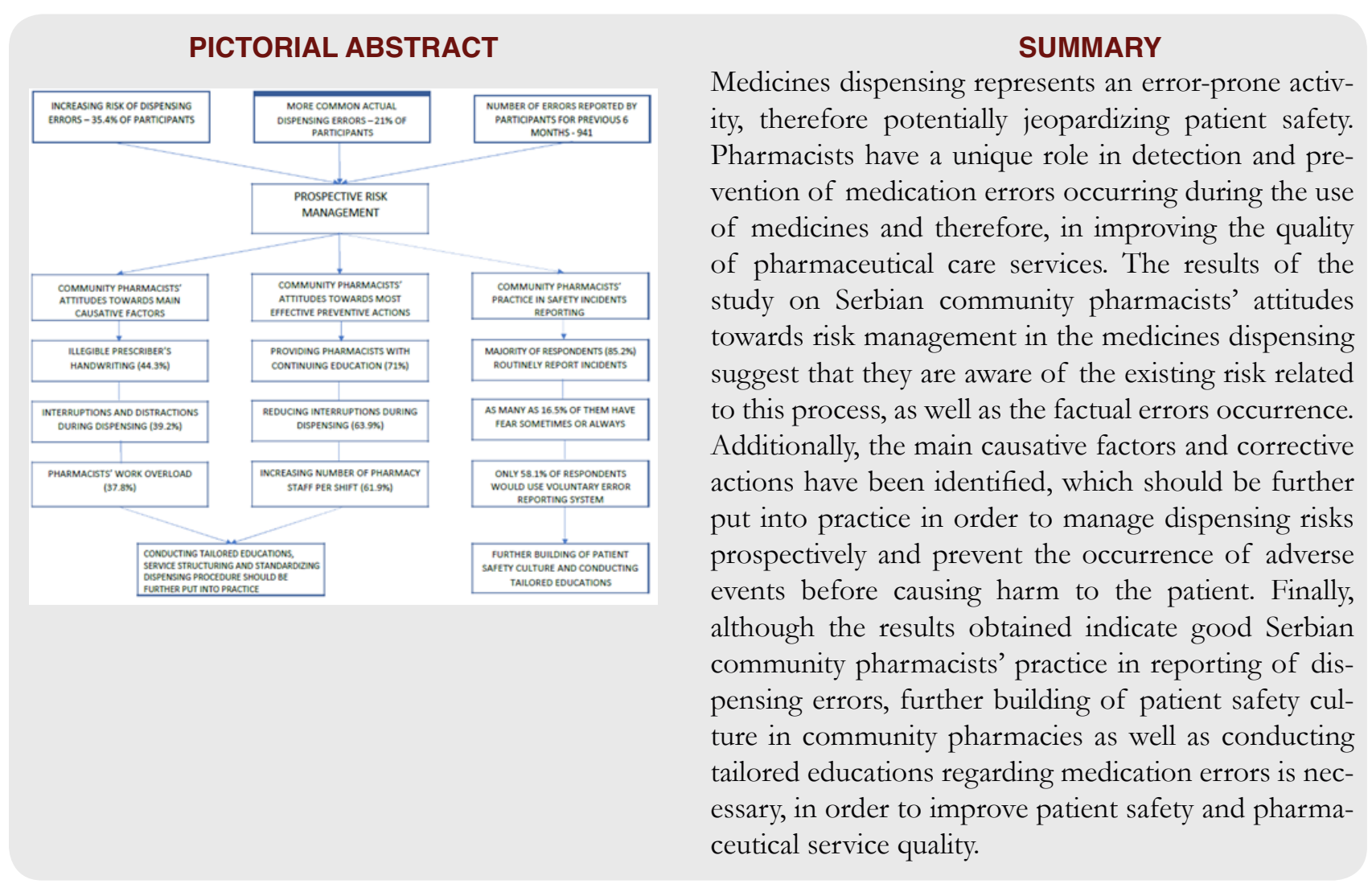

30. Schneider PJ, Sill BE. Education and training to provide pharmaceutical care. International Pharmacy Journal. 1995;9(4):156-60.

31. Currie JD, Chrischilles EA, Kuehl AK, Buser RA. Effect of a training program on community pharmacists' detection of and intervention in drug-related problems. J Am Pharm Assoc. 1997;37(2):182-91.

32. Baker A. Crossing the quality chasm: A new health system for the $21^{\text {st }}$ century. BMJ. 2001;323(7322):1192.

33. Airaksinen M. The Role of communication skills: Developing patient centred practice in community pharmacies. The Chronic III. 2004;8:10-4.

34. Varadarajan R, Barker KN, Flynn EA, Thomas RE. Comparison of two errordetection methods in a mail service pharmacy serving health facilities. J Am Pharm Assoc. 2008;48(3):371-82a.

35. Kelly WN. Medication errors: Lessons learned and actions needed. Prof Saf. 2004;49(7):35.

36. Ashcroft DM, Morecroft C, Parker D, Noyce PR. Likelihood of reporting adverse events in community pharmacy: An experimental study. Qual Saf Health Care. 2006;15(1):48-52.

37. Boyle TA, Mahaffey T, MacKinnon NJ, Deal H, Hallstrom LK, Morgan $H$. Determinants of medication incident reporting, recovery and learning in community pharmacies: A conceptual model. Res Social Adm Pharm. 2011;7(1):93-107.

38. Stump LS. Re-engineering the medication error-reporting process: Removing the blame and improving the system. Am J Health Syst Pharm. 2000;57(Suppl 4):S10-7.

39. Ashcroft DM, Morecroft C, Parker D, Noyce PR. Safety culture assessment in community pharmacy: development, face validity and feasibility of the Manchester Patient Safety Assessment Framework. BMJ Qual Saf. 2005;14(6):417-21.

40. Institute for Safe Medication Practices. Medication Safety Self-Assessment for Community/Ambulatory Pharmacy. 2017. [cited 2019 March 23]. Available from: https://www.ismp.org/assessments/community-ambulatory-pharmacy. 


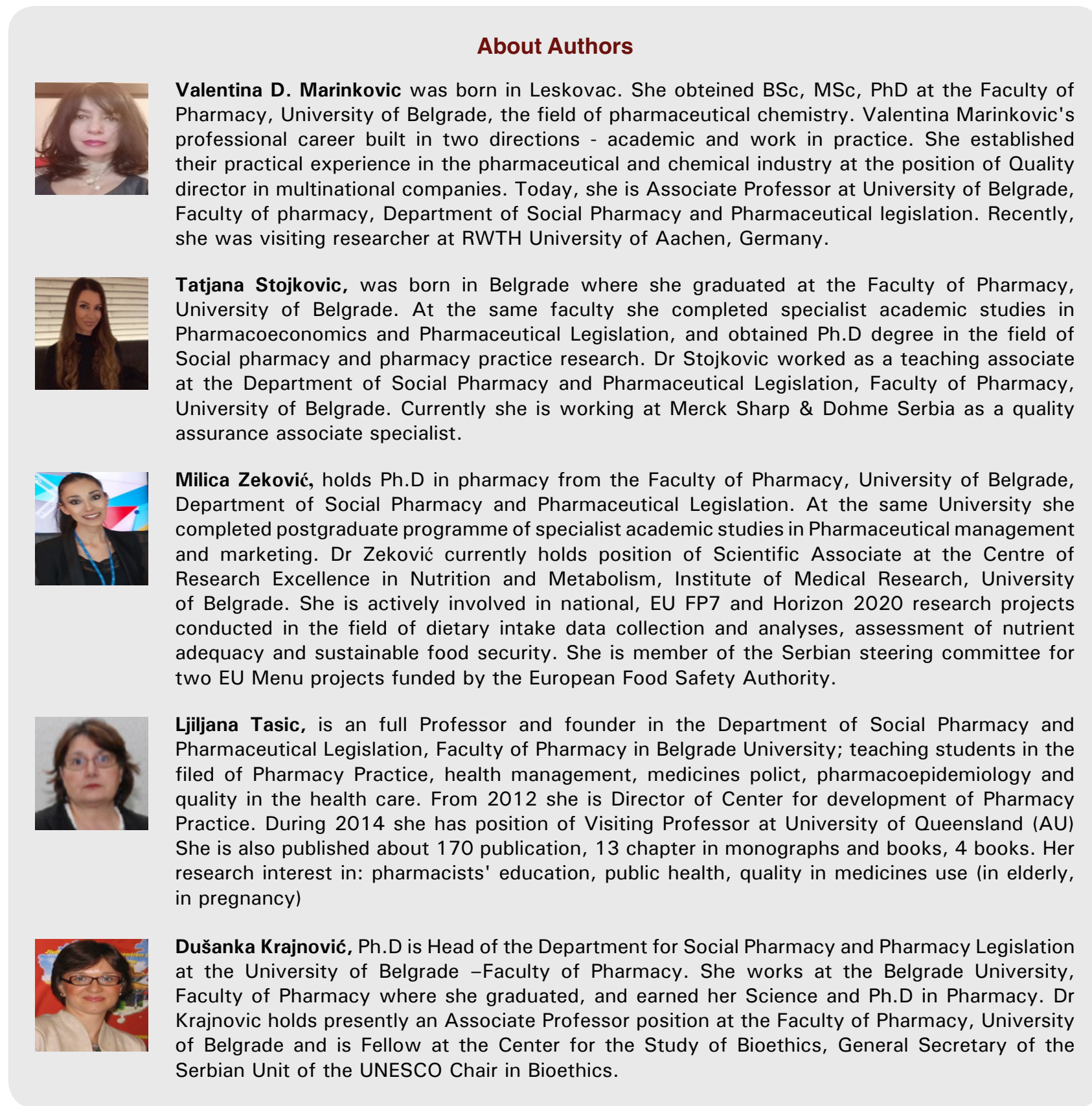

Cite this article: Marinkovic V, Stojkovic T, Zekovic M, Tasic L, Krajnovic D. Community Pharmacists' Attitudes and Professional Practice in Relation to the Patient Safety Incidents. Indian J of Pharmaceutical Education and Research. 2020;54(1):194-205. 